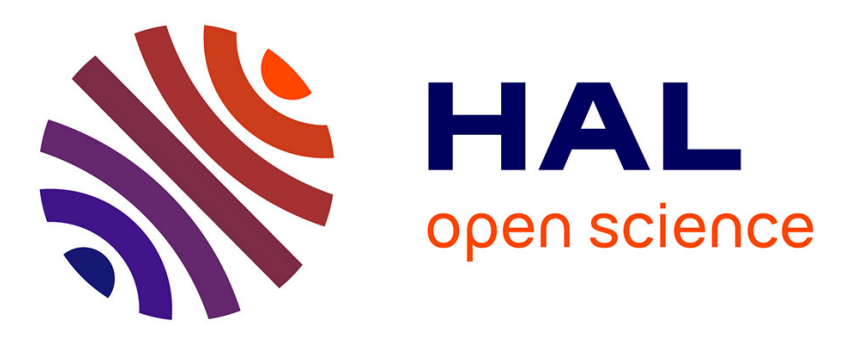

\title{
The Concept of the Machining Surface in 5-Axis Milling of Free-form Surfaces
}

Christophe Tournier, Emmanuel Duc, Claire Lartigue, Alexandre Contri

\section{To cite this version:}

Christophe Tournier, Emmanuel Duc, Claire Lartigue, Alexandre Contri. The Concept of the Machining Surface in 5-Axis Milling of Free-form Surfaces. 3nd International Conference on Integrated Design and Manufacturing In Mechanical Engineering, May 2000, Montreal, Canada. pp.279-286, 10.1007/978-94-015-9966-5_33. hal-01223061

\section{HAL Id: hal-01223061 https://hal.science/hal-01223061}

Submitted on 2 Nov 2015

HAL is a multi-disciplinary open access archive for the deposit and dissemination of scientific research documents, whether they are published or not. The documents may come from teaching and research institutions in France or abroad, or from public or private research centers.
L'archive ouverte pluridisciplinaire HAL, est destinée au dépôt et à la diffusion de documents scientifiques de niveau recherche, publiés ou non, émanant des établissements d'enseignement et de recherche français ou étrangers, des laboratoires publics ou privés. 


\title{
The concept of the machining surface in 5-axis milling of free-form surfaces
}

\author{
Christophe Tournier \\ Laboratoire Universitaire de Recherche en Production Automatisée \\ ENS de Cachan \\ email : tournier@1urpa.ens-cachan.fr \\ Emmanuel Duc \\ Lurpa - Ens de Cachan \\ Claire Lartigue \\ Lurpa - Ens de Cachan \\ Alexandre Contri \\ Lurpa - Ens de Cachan
}

\begin{abstract}
The concept of the machining surface $(M S)$ is a new approach to the process of design and manufacturing of free form surfaces. The machining surface is the surface representation of the tool path, integrating functional design specifications and machining constraints. By definition, the machining surface is a surface including all the information necessary for the driving of the tool, so that the envelope surface of the tool movement sweeping the $M S$ gives the expected free-form. In this paper, we study the building of the $M S$ for 5-axis end milling with usual cutting tools, ball, flat and filleted endmill. We make so that the design and manufacturing constraints taken into account by the machining surface are completely uncoupled within the $M S$.
\end{abstract}

Résumé : Le concept de la surface d'usinage apporte une évolution dans le processus de réalisation des pièces de forme gauche au niveau de la conception et de la fabrication. La surface d'usinage est une représentation surfacique du trajet de l'outil intégrant les contraintes fonctionnelles de conception ainsi que les contraintes technologiques de fabrication. Par définition, la surface d'usinage guide un point fixe de l'outil de telle sorte que la surface enveloppe du mouvement de l'outil soit la surface attendue. Nous présentons dans cet article comment se construit la surface d'usinage pour le fraisage à cinq axes en bout avec les outils de coupe couramment utilisés. Nous faisons en sorte que les aspects conception et fa- brication pris en compte par la surface d'usinage soient totalement découplés afin de conserver ces activités indépendantes.

Introduction : The elaboration process of free-form surfaces must ensure the adequacy between the design intent and the produced part. The process consists in two main activities : the engineering and the manufacturing process (Fig. 1). The engineering process starts with styling and includes engineering constraints through the definition of high quality surfaces in the CAD database. The CAD model becomes the common support from the digital mock-up to the digital manufacturing. Then the CAM system computes the tool path to machine the part or its shape in a mould or a die. Finally, the machined part results from the envelope of the tool motion, i.e. the envelope of the calculated tool path.

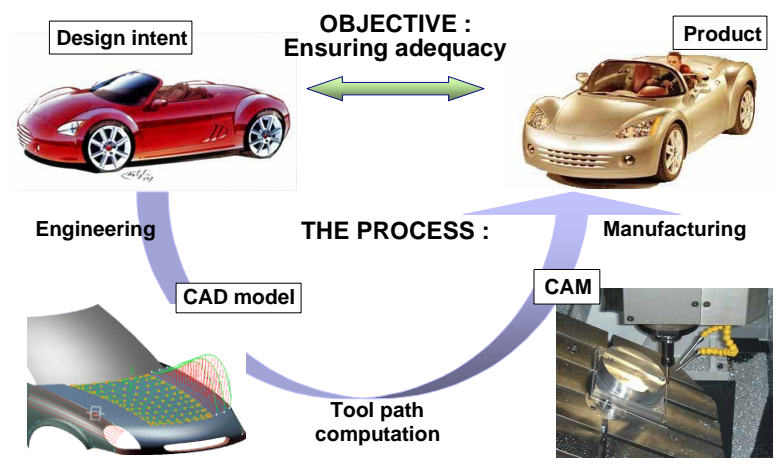

Figure 1 : Elaboration process of free-form surfaces

Each link of the process chain is liable to introduce errors between the final part and the 
design intent. First of all there may be a deterioration of data when functional requirement and design intent are converted into tridimensionnal CAD data. In spite of the continuous improvement of CAD modeler, the techniques of surface construction remain limited. It is not always possible to obtain the forms expected by the designer as well as continuity connections between surfaces that are necessary to a precise machining. User's actions depend on the CAD software functionalities and the resulting shape does not fit with the user intent but is the closest shape the modeler can produce. Furthermore, styling or engineering specifications are lost, only the CAD model describes geometry for downstream applications.

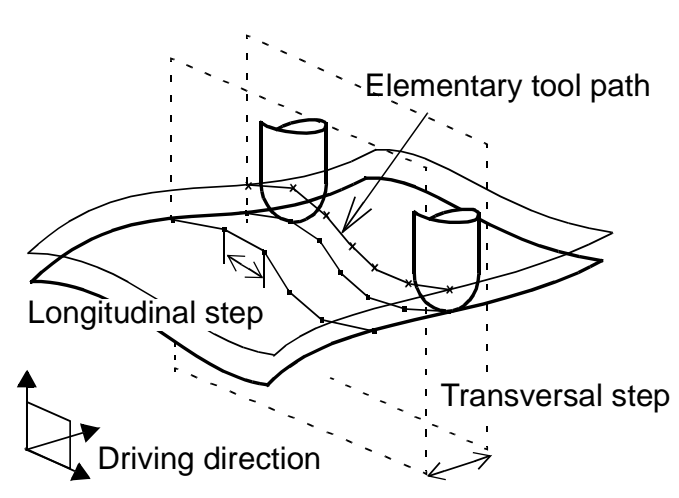

Figure 2 : Tool path generation

In the process, the tool path computation and the machining activities must guarantee a part that meets the geometry of the CAD model. But other errors are introduced during the tool path computation and during the machining because of the dynamic behaviour of the machine tool.

The tool path generation for sculptured surfaces in 3 or 5 -axis end milling relies on the choice of a tool driving direction and two discretization steps, the step length along the path (longitudinal step) and the cutter path interval (transversal step) (Fig. 2). The values of the discretization steps must be linked to the geometrical specifications of form deviation or roughness. They must also ensure the achievement of sharp edges and curvatures radii specified on the part.

The concept of the machining surface has been developed in order to ensure the accura- cy between the machined part and the design intent and to optimize the tool path planning [2]. We present in this paper the result of our work concerning the use of the machining surface for the tool path planning in 5-axis milling. Our previous work on the adequacy between the machined part and the design intent has been presented in [1].

\section{The concept of the machining surface :}

The machining surface (MS) is a surface including all the information necessary for the driving of the tool, so that the envelope surface of the tool movement sweeping the MS gives the expected free-form. (Fig. 3).

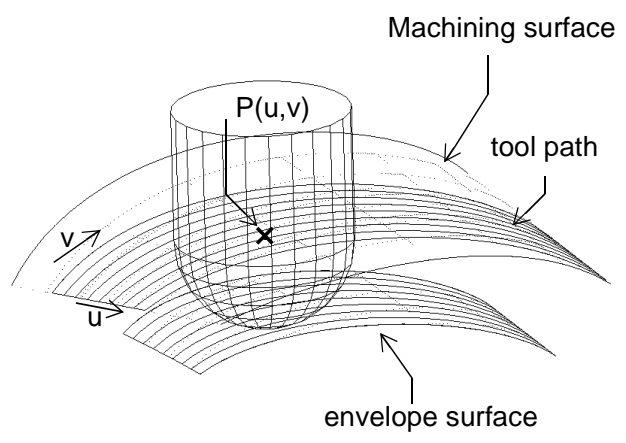

Figure 3 : The machining surface

The MS is built on geometrical elements so that the envelope surface respects the geometrical constraints of the design intent. The machining surface is the surface representation of all the cutter location, as the offset surface gathers centre point location of a ball endmill cutter. Then the tool path planning consists in the choice of curves on the machining surface (Fig. 4).

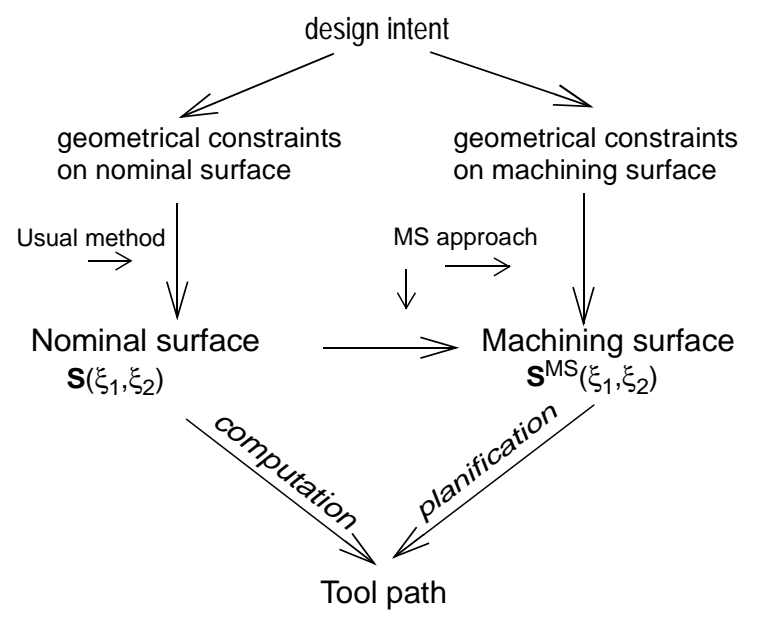

Figure 4 : Evolution of the process 
In usual methods of free-form design and machining, the geometrical constraints are respected by the nominal surface. The tool path is planned on the nominal surface. This is the trajectory of the contact point between the surface and the tool, the $C_{C}$ point (cutter contact). The sampling of the path according to the step length criteria produces successive $C_{C}$ point locations. Then, the center point of the tool $C_{L}$ (cutter location), which is used by the numerical controller during machining, is computed regarding to the tool geometry (Fig. 5). The tool path computation based on $C_{C}$ points generally produces errors such as the error in the respect of the chordal deviation when using the linear interpolation [4] or oscillations when using Nurbs interpolation [2].

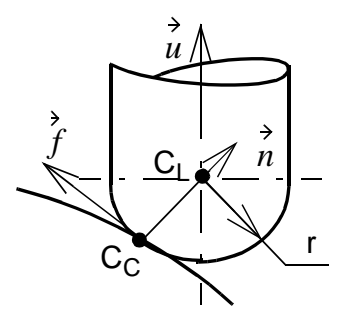

$$
C_{L}=C_{C}+r \cdot \vec{n}
$$

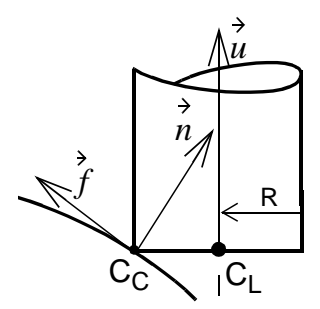

$$
\begin{aligned}
& C_{L}=C_{C}+R \cdot \vec{v} \\
& \vec{v}=\frac{\vec{u} \wedge \vec{n}}{\|\vec{u} \wedge \vec{n}\|}
\end{aligned}
$$

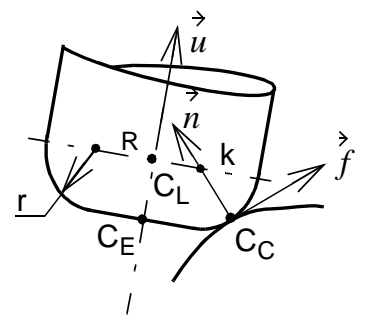

$$
\begin{aligned}
& C_{L}=C_{C}+r \cdot \vec{n}+R \cdot \vec{v} \\
& \vec{v}=\frac{\vec{u} \wedge \vec{n}}{\|\vec{u} \wedge \vec{n}\|} \wedge \vec{u}
\end{aligned}
$$

Figure 5 : Tools geometry

The sampling phase from the nominal surface to the set of discret $C_{L}$ points generates geometric deviations between the envelope of the tool movement and the expected surface [3]. The two-dimensional and continous approach suggested by the machining surface helps to avoid the problems previously mentioned. Thus the $M S$ allows the planning of successive and adjacent $C_{L}$ points to create the tool path.

Our objective is to build a surface on which we can compute curves as tool paths, according to a machining strategy. The shape of the machining surface must lead to the respect of the design intent, whatever the machining strategy adopted. In 5-axis milling, the machining surface supports the positioning of the effective cutting area of the tool and the orientation of the tool axis.

5-axis end milling toolpath generation : The toolpath generation in 5-axis milling consists in the computation of the cutter center location $C_{L}$ and the tool axis vector $\vec{u}$ for each point of contact $C_{C}$ between the tool and the surface along the tool path [5][6].

To ensure the tangency between the tool and the machined surface and avoid gouging, the tool can be rotated around the two vectors $\vec{t}$ and $\vec{n}$ of the local coordinate frame defined by : $\left(C_{G}, \vec{f}, \vec{n}, \vec{t}\right)$ where : $\vec{f}$ is the tool feed vector, $n$ the vector normal to the surface and $\vec{t}$ the vector tangent to the surface with $\vec{t}=\vec{f} \wedge \vec{n}$

Initially, the tool is positioned onto the surface at $C_{C}$ so that the axis vector $\vec{u}$ is parallel to the normal vector $\vec{n}$. Then we apply the first rotation $\Omega_{\mathrm{t}}$ around the vector $\vec{t} t$ and second rotation $\Omega_{\mathrm{n}}$ around the vector $\vec{n}$ (Fig. 6) [5].

Nevertheless, the movement of rotation is not centered at the same point in function of the tool geometry. For the flat endmill, the rotation $\Omega_{\mathrm{t}}$ is applied around the line $\left(C_{\vec{C}}, \vec{t}\right)$ and the rotation $\Omega_{\mathrm{n}}$ around the line $\left(C_{C}, \overrightarrow{\vec{n}}\right)$. On the other hand, for the filleted endmill the rotation $\Omega_{\mathrm{t}}$ must be applied arround the line $(K, \vec{t})$ to prevent the tool from rolling on the surface and keep it tangent to the surface at $C_{C}$. The second rotation $\Omega_{\mathrm{n}}$ is applied around the line $\left(C_{C}, \vec{n}\right)$ passing though the point $K$. So, $K$ is considered as the instantaneous rotation center of the two rotations. This remark remains valid with the ball endmill that can be considered as a filleted endmill with a principal radius $R$ equal to zero. 

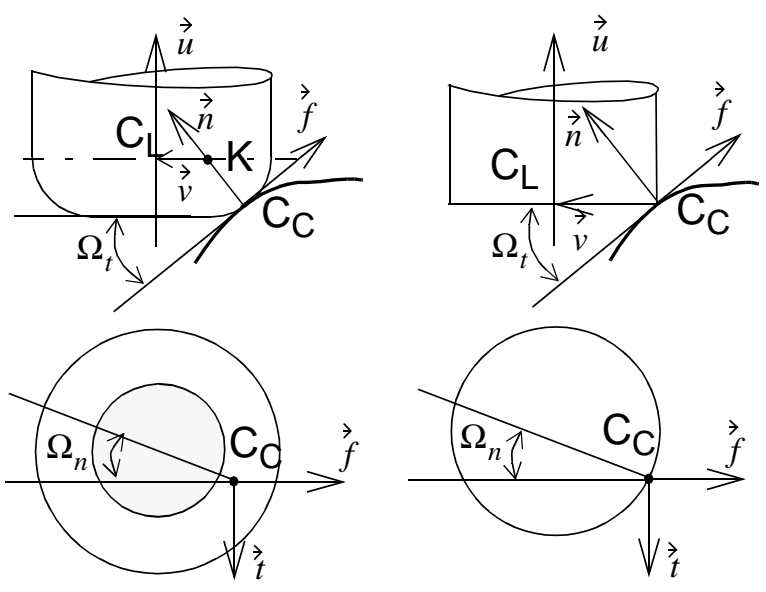

Figure 6 : Tool positioning in 5-axis milling

Creation of the machining surface : Our objective is to determine the machining surface according to the geometry of the tool and the number of axes used. We remind that the machining surface is the locus of points belonging to the tool, allowing its setting in position for each moment and in a unique way whatever the machined point. The machining surface may consist of one or more different surfaces.

First of all, we show that the couple $\left(C_{L}, \vec{u}\right)$ used conventionally to locate the tool in 5-axis is not appropriate to conceive the machined surface in the case of the filleted and the flat tools. Indeed, the orientation of the tool axis $\vec{u}$ is defined in a local coordinate system related to the normal of the surface $\vec{n}$ and the cutter feed direction $\vec{f}$. That means that the cutter feed direction, therefore the machining strategy, is known during the generation of the successive tool positions. The resulting machined surface, defined as the locus of $C_{L}$ and $P$ so that $\overrightarrow{C_{L} P}=\vec{u}$, would then be a particular case adapted to the adopted machining strategy. Therefore, it becomes not possible to uncouple the design and manufacture phases. The choice of the couple $\left(C_{L}, \vec{u}\right)$ is then not appropriate for the filleted and flat endmills.

On the other hand for the ball endmill, the position of the center point $C_{L}$ is independent of the tool axis orientation. Thus, we preserve the couple $\left(C_{L}, \vec{u}\right)$ to locate the ball endmill tool. We seek for another way of positioning the flat and the filleted endmills. In order to deal with a general case, let us consider the 5-axis milling with a filleted endmill then we will extrapolate the results to the other tool geometries. Rather than take the tool center $C_{L}$ and its axis $\vec{u}$ to position the tool, we suggest to use the point $K$ and the vector $\vec{v}=\overrightarrow{K C_{L}}$ (Fig. 6). $K$ is defined as the offset point of $C_{C}$ with an offset distance equal to the corner radius $r$ of the tool. The point $K$ plays a particular role because it belongs to the central axis of the torsor associated to the movement of the tool relatively to the nominal surface. Thus, $K$ remains fixed during the rotational movements of the tool when the tool is set in position. There remains a possible rotation around the vector $\vec{v}$. However, it should be noticed that the tool axis vector $\vec{u}$, the vector $\vec{v}$ and the normal vector $\vec{n}$ passing through $C_{C}$ remain always coplanar during the two rotations $\Omega_{\mathrm{t}}$ and $\Omega_{\mathrm{n}}$. Indeed, they are coplanar since the beginning of the setting in position because at any point $C_{C}$, the normal to the surface passes by the axis of the torus (cf Appendix). Then, the two rotations around $\vec{t}$ and $\vec{n}$ leave the vectors $\vec{u}, \vec{v}, \vec{n}$ coplanar.

Knowing points $K, \mathrm{C}_{L}$ and the normal vector $\vec{n}$ is sufficient to position the tool in the $3 \mathrm{D}$ space. The tool axis vector is then defined by :

$\vec{u}=\vec{v} \wedge \frac{\vec{n} \wedge \vec{v}}{\|\vec{n} \wedge \vec{v}\|}$ with $\vec{v}=\overrightarrow{K C_{L}}$

The points $K$ and $C_{L}$ make it possible to define a unique tool position because they are located in the symmetry plane of the tool.

The machining surface is thus composed of two surfaces $S_{1}$ and $S_{2}$, loci of the points $K$ and $C_{L}$ (Fig. 7). The normal vector is embedded in the equation of $S_{l}$. We call the surface $S_{1}$ the guiding surface and surface $S_{2}$ the orientation surface. The guiding surface $S_{1}$ is the offset surface of the nominal surface with magnitude equal to the corner radius $r$ of the tool. It is thus independent of the machining strategy. The orientation surface $S_{2}$ is the surface which gives the orientation of the tool axis according to the considered machining strategy. 


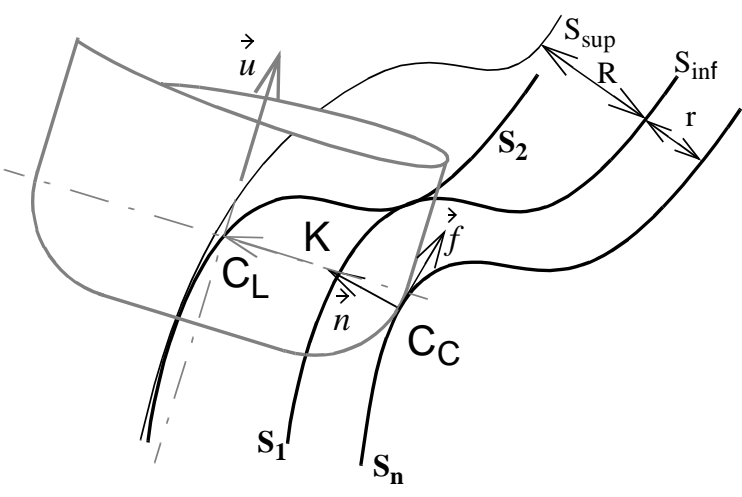

Figure $7: M S$ in 5-axis milling with a filleted endmill

Conditions : The orientation surface $S_{2}$ is located between two limit surfaces $S_{\text {inf }}$ and $S_{\text {sup }}$. From the parametric equation $\Phi\left(\xi^{1}, \xi^{2}\right)$ of the normal surface $S_{n}$, we can write :

$\Phi_{\text {sup }}\left(\xi^{1}, \xi^{2}\right)=\Phi\left(\xi^{1}, \xi^{2}\right)+(r+R) \cdot N\left(\xi^{1}, \xi^{2}\right)$

The upper limit corresponds to the case for which the tool axis orientation $\vec{u}$ is parallel to the tool feed direction $f$. Moreover, for a given tool path $\left(C_{1}\right)$ on surface $S_{1}$, the associated curve $\left(C_{2}\right)$ on surface $S_{2}$ is included in the pipe surface of radius $R$ and of spine curve $\left(C_{1}\right)$. This condition materializes the fixed distance between $K$ and $C_{L}$.

$\Phi_{i n f}\left(\xi^{1}, \xi^{2}\right)=\Phi\left(\xi^{1}, \xi^{2}\right)+r \cdot \vec{n}\left(\xi^{1}, \xi^{2}\right)$

The lower limit corresponds to the case for which the tool axis orientation $\vec{u}$ is parallel to the normal vector $\vec{n}$. This lower limit is a condition necessary but not sufficient to avoid gouging.

Other tool geometries : The results obtained in the case of 5-axis milling with a filleted endmill can be extended to the other tool geometries. The flat endmill can be regarded as a filleted endmill with a radius of corner $r$ null. The point $K$ coincides then with the cutter contact point $C_{C}$ and the surface $S_{l}$, locus of the points $K$ is the nominal surface to be machined. In this case, the machining strategy controls the cutter contact point. As previous- ly exposed, the ball endmill can be considered as a filleted endmill with a principal radius $R$ equal to zero. The point $K$ coincides then with the point $C_{L}$. The adopted solution that uses the points $K$ and $C_{L}$ is not valid for this type of tool. Thus, we use the original configuration with the parameters $C_{L}$ and $\vec{u}$.

According to the tool geometry, the machining surface breaks up into two distinct surfaces : the guiding surface $S_{l}$ which is the offset surface of the nominal surface, and the surface of orientation $S_{2}$ whose shape depends on the machining strategy.

\section{Construction of the guiding and orientation}

surfaces : Let us consider the nominal surface $S_{n}$ of $R^{3}$ and $F$ the map which transforms an interval of the parametric plane $\left(\mathrm{O}, \xi^{1}, \xi^{2}\right)$ into $S_{n}$. From nominal surface $S_{n}$ described by $F\left(\xi^{1}, \xi^{2}\right)$ one can determine the map of the guiding surface: $\Phi^{g u i}\left(\xi^{1}, \xi^{2}\right)$ :

$$
\Phi^{g u i}\left(\xi^{1}, \xi^{2}\right)=F\left(\xi^{1}, \xi^{2}\right)+r \vec{n}\left(\xi^{1}, \xi^{2}\right)
$$

The orientation surface is built according to the orientation we wish to give to the tool along the tool path.

The orientation of the tool axis is described by $\vec{u}\left(\xi^{1}, \xi^{2}\right)$. One can evaluate the map of the orientation surface $\Phi^{\text {ori }}\left(\xi^{1}, \xi^{2}\right)$ followed by the center of various types of tools :

for the ball end mill of radius $r$ :

$\Phi^{\text {ori }}\left(\xi^{1}, \xi^{2}\right)=F\left(\xi^{1}, \xi^{2}\right)+r \overrightarrow{r n}\left(\xi^{1}, \xi^{2}\right)+\vec{u}\left(\xi^{1}, \xi^{2}\right)$

for the flat end mill of radius $R$ :

$$
\Phi^{\text {ori }}\left(\xi^{1}, \xi^{2}\right)=F\left(\xi^{1}, \xi^{2}\right)+R \vec{v}\left(\xi^{1}, \xi^{2}\right)
$$

for the filleted end mill of radii $R$ and $r$ :

$$
\begin{aligned}
& \Phi^{\text {ori }}\left(\xi^{1}, \xi^{2}\right)=F\left(\xi^{1}, \xi^{2}\right)+\overrightarrow{r n}\left(\xi^{1}, \xi^{2}\right)+R \vec{v}\left(\xi^{1}, \xi^{2}\right) \\
& \text { with } \vec{v}=\frac{\vec{u} \wedge \vec{n}}{\|\vec{u} \wedge \vec{n}\|} \wedge \vec{u}
\end{aligned}
$$

The following table gathers surfaces to be regarded as machining surface according to the type of tool and the number of axes used for machining. 
Table 1: Various machining surfaces

\begin{tabular}{|c|c|c|}
\hline & 3-axis & 5-axis \\
\hline $\begin{array}{l}\text { Ball } \\
\text { endmill }\end{array}$ & $S_{1}: \boldsymbol{F}+r \vec{n}$ & $\begin{array}{l}S_{1}: \boldsymbol{F}+r \vec{n} \\
S_{2}: \boldsymbol{F}+r \vec{n}+\vec{u}\end{array}$ \\
\hline $\begin{array}{l}\text { Flat } \\
\text { endmill }\end{array}$ & $S_{1}: \boldsymbol{F}+R \vec{v}$ & $\begin{array}{l}S_{1}: \boldsymbol{F} \\
S_{2}: \boldsymbol{F}+R \vec{v}\end{array}$ \\
\hline $\begin{array}{l}\text { Filleted } \\
\text { endmill }\end{array}$ & $S_{1}: \boldsymbol{F}+r \vec{n}+R \vec{v}$ & $\begin{array}{l}S_{1}: \boldsymbol{F}+\overrightarrow{r n} \\
S_{2}: \boldsymbol{F}+\overrightarrow{r n}+R \vec{v}\end{array}$ \\
\hline
\end{tabular}

We propose to build the guiding and orientation surfaces according to the method described in [7] because $S_{1}$ and $S_{2}$ are generalised offset surfaces. $S_{1}$ and $S_{2}$ are modeled by a multi-patch surface. Patches are bi-cubic interpolants connected in tangency. Each patch is expressed as a Ferguson tensor product surface :

$$
r(u, v)=U C Q C^{T} V^{T}
$$

with $C$ the matrix of blending function (cubic Hermite basis) and $Q$ the Coons matrix [8]:

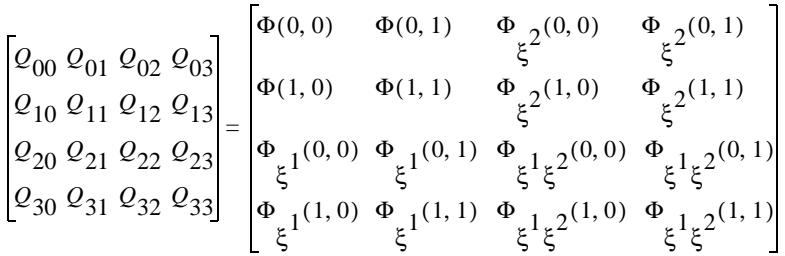

where $\Phi\left(\xi^{1}, \xi^{2}\right)$ is the implicit equation of the guiding or orientation surface. In order to keep the consistency of the surface representation in the CAD system, we use a Bezier (UNISURF) representation for patches :

$$
r(u, v)=U M B M^{T} V^{T}
$$

with $M$ the cubic Bernstein basis matrix and $B$ the matrix of the Characteristic Polyhedron vertices expressed as :

$$
B=\left(M^{-1} C\right) Q\left(M^{-1} C\right)^{T}
$$

The precision obtained on surfaces $S_{1}$ and $S_{2}$ is proportional to the number of patches. From a point $K$ on $S_{1}$ one finds the corresponding point $C_{L}$ on $S_{2}$ with the assumption that parameter setting of $S_{1}$ and $S_{2}$ are identical.

Thus the method generates approximations. The errors on the guiding surface $S_{l}$ must be controlled because they cause local gouging between the tool and the nominal surface at the $C_{C}$ point. We have to use a large number of patches in the offset surface approximation to perform a precise machining. On the other hand, errors on the orientation surface $S_{2}$ present fewer disadvantages because the variations of orientation of the tool axis do not generate machining errors. Indeed, the controlled point is the point $K$ which is the instantaneous center of rotation during two rotations of the tool. The orientation of $\vec{u}$ does not influence the position of the active part of the tool. Moreover the tool radius $R$ reduces the amplitude of the variations of $S_{2}$ on the orientation of the axis tool (Fig. 8). The maximum angle variation is given by :

$$
\tan \alpha_{\text {max }}=\frac{\varepsilon}{R}
$$

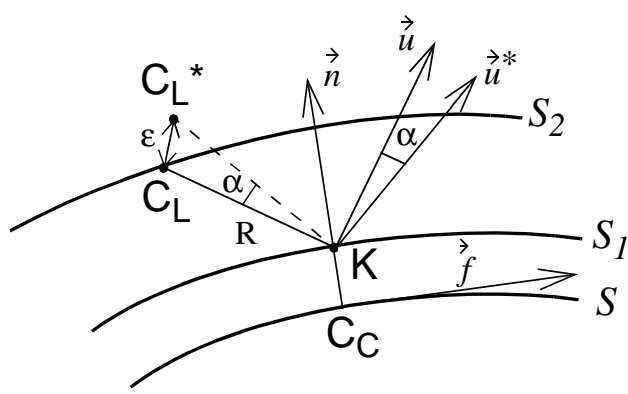

Figure 8 : Variation of tool axis orientation

In conventional generation of tool path with a filleted endmill, the rotation $\Omega_{\mathrm{t}}$ is done around $\left(C_{C}, \vec{t}\right)$ [6]. The $C_{C}$ point is then the instantaneous center of rotation. Since $C_{C}$ is the contact point between the tool and the surface, the tool is rolling on the surface at $C_{C}$. If there is an error in the calculation of the orientation of the tool axis, a collision appears at the $C_{C}$ point.

Example : The treated example consists in machining a surface (a Bezier patch of degree $5)$ with a filleted endmill $(R=10, r=4)$ and 
with the following strategy: 5-axes milling, tool center guiding along parallel planes to yoz and $\Omega_{\mathrm{t}}=20^{\circ}, \Omega_{\mathrm{n}}=0^{\circ}$. $S_{l}$ is the offset surface of $S_{n}$ with an offset distance equal to 4 $\mathrm{mm} . S_{2}$ is the generalised offset surface $\left(S_{2}=S_{n}+r \vec{n}+R \overrightarrow{\boldsymbol{v}}\right)$ with offset distance equal to $R=10$ and $r=4$. $S_{1}$ and $S_{2}$ are free from loops. The modeling of the kinematics of the displacement of the tool on both surfaces $S_{1}$ and $S_{2}$ made it possible to simulate the machining along the curve $C_{l}$ (Fig. 9), intersection of $S_{l}$ with the guiding plane. $C$ is the contact curve followed by the $C_{C}$ point on the nominal surface and $C_{2}$ is the curve followed by $C_{L}$ on the orientation surface.

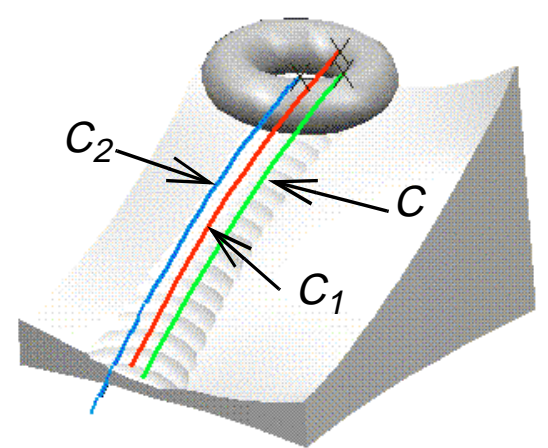

Figure 9 : Machining simulation

Concluding remarks : The concept of the machining surface offers a new solution to generate toolpaths. Its construction is common to the various cutting tools usually used. The guiding surface creation is only based on the corner radius of the considered tool, independently of the machining strategy. The planning of the tool paths is carried out initially by choosing curves on the guiding surface. Then if necessary, the surface of orientation makes it possible to position the tool for machining with five axes.

The objective is now to use the machining surface to generate successive tool paths on a compound surface. To achieve this task, we will have to introduce technical surfaces in the machining surface framework to be able to add approach, retract and linking macros.

We are also working on another method to build the orientation and guiding surface. The method would be based on the two fundamental forms to evaluate the local characteristics of the orientation and guiding surfaces.
Indeed, the current approach is difficult to implement. The encountered difficulties come from several factors : the loops resulting from the offset operation, the need for synchronizing the parameter settings, the number of patches required to achieve hight quality machining.

\section{References :}

[1] E. Duc, C. Lartigue, C. Tournier, P. Bourdet, A new concept for the design and the manufacturing offree-form surfaces: the machining surface. Annals of the CIRP - 1999, vol 48/1, 103-106.

[2] E. Duc, Usinages des formes gauches, contribution à l'amélioration de la qualité des trajectoires d'usinage. Thèse de Doctorat ENS Cachan, 1998.

[3] C. Lartigue, E. Duc, C. Tournier, Machining of free-form surfaces and geometrical specifications, IMechE 1999, Proceedings of the Institution of Mechanical Engineers Vol. 213 Part B, 21-27

[4] B.K. Choi, C.S. Lee, J.S Hwang, C.S. Jun, Compound surface modelling and machining, Computer Aided Design, 1988, vol 20, no 30, 127-136.

[5] B.K. Choi, J.W. Park, C.S. Jun, Cutter location data optimization in 5-axis surface machining, Computer aided Design, 1993, vol 25, no 6, 377-386.

[6] Y.S Lee, Admissible tool orientation control of gouging avoidance for 5-axis complex surface machining, Computer Aided Design, 1997, vol 29, no 7, 507-521.

[7] R.T. Farouki, The approximation of non degenerate offset surfaces, Computer Aided Geometric Design, 1986, no 3, 15-43.

[8] I.D. Faux, M.J. Pratt, Computional Geometry for Design and Manufacture, 1979, Ellis Horwood Ltd, Chistester. 


\section{Appendix :}

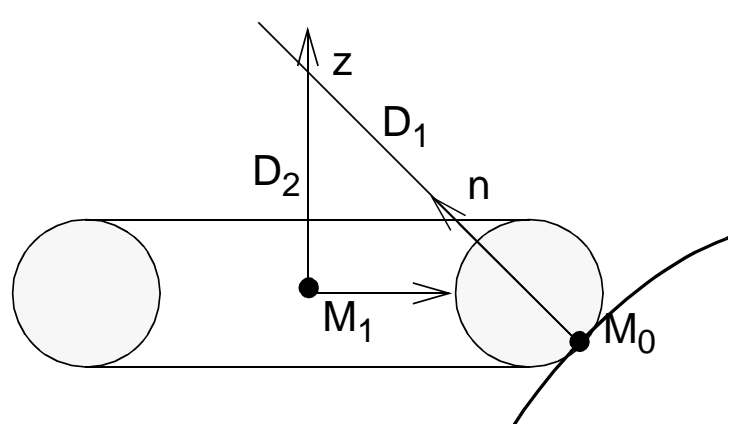

Figure 10 : Torus geometry

Implicit equation of the torus :

$S(x, y, z)=\left(x^{2}+y^{2}+z^{2}+d^{2}-r^{2}\right)^{2}-4 d^{2}\left(x^{2}+y^{2}\right)$

Normal vector to the torus at $M_{0}$ :

$$
\left\{\begin{array}{l}
n_{x} \\
n_{y} \\
n_{z}
\end{array}=\left\{\begin{array}{l}
S_{x}=4 x_{0}\left(x_{0}^{2}+y_{0}^{2}+z_{0}^{2}+d^{2}-r^{2}\right)-8 d^{2} x \\
S_{y}^{\prime}=4 y_{0}\left(x_{0}^{2}+y_{0}^{2}+z_{0}^{2}+d^{2}-r^{2}\right)-8 d^{2} y \\
S_{z}^{\prime}=4 z_{0}\left(x_{0}^{2}+y_{0}^{2}+z_{0}^{2}+d^{2}-r^{2}\right)
\end{array}\right.\right.
$$

Let $D_{1}$ and $D_{2}$ be the two lines passing through the normal vector and the tool axis vector :

$\begin{cases}D_{1} & \overrightarrow{O M}=\overrightarrow{O M_{0}}+\mu \cdot \vec{n} \\ D_{2} & \overrightarrow{O M}=\overrightarrow{O M_{1}}+\lambda \cdot \vec{z}\end{cases}$

The distance between the two lines is $d$ :

$$
d=\left|\frac{\left(\overrightarrow{O M_{0}}-\overrightarrow{O M_{1}}\right) \cdot(\vec{n} \wedge \vec{z})}{|(\vec{n} \wedge \vec{z})|}\right|
$$

If $M_{1}$ is the origin of the coordinate system, we find :

$$
\overrightarrow{O M_{0}} \cdot(\vec{n} \wedge \vec{z})=\left(x_{0} \vec{i}+y_{0} \vec{j}+z_{0} \vec{k}\right) \cdot\left(n_{y} \cdot \vec{i}-n_{x} \cdot \vec{j}\right)
$$

whatever the location of the point $M_{O}$ on the torus. The two lines $D 1$ and $D 2$ intersect. 